\title{
A Decision Support System of Quality Forecast Evaluation for Complex Products Based on Data Warehouse
}

\author{
Jihong Pang ${ }^{1,}$ a * \\ ${ }^{1}$ College of Mechanical and Electronic Engineering, Wenzhou University, Wenzhou 325035, \\ Zhejiang, China \\ aemail:pangjihong@163.com
}

\begin{abstract}
Keywords: Complex Products, Product Quality, Quality Forecast, Decision Support System, Data Warehouse
\end{abstract}

Abstract. How to improve the quality of complex products was a difficult problem to most enterprises. It is the most important to increase product quality by establishing effective forecasting methods. This article presents the findings of a decision support system (DSS) of quality forecast evaluation for complex products based on data warehouse. Firstly, the implementation framework for DSS based on data warehouse is presented. Then, a new decision method based on data processing mining is introduced. Finally, a case study is presented to shown the approved methods and models by using an example of computer numerical control (CNC) machines in practice implementations. Simulation results of this model applied to DSS show its validity.

\section{Introduction}

In recent years, competition and performance expectations among manufacturing companies have increased dramatically. So most enterprises have recognized the quality problem and were determined to improve it. Many companies' prosperity depends on producing better goods. An excellent product is getting a solid reputation for having top of the line products. Higher-quality product also lays the fundamental answer to better economic efficiency and competitiveness[1]. Thus, good companies must make ceaseless efforts to improve the quality of their products. And a lot of enterprises have potentials to improve their management expertise, product quality and service to meet customer needs[2]. On the other hand, companies need more feedback from the consumer and quality control processes in order to improve their goods. The company manager's concern was how to improve the quality of their products[3]. In line with coexist principle of customer needs, require with customer for let already, quality consciousness deepen company each staff to improve the management level of product quality further. Furthermore, management requires measurement of product quality, product innovation, and customer satisfaction[4]. They must focus on improving the quality of their products. In a word, the goal of this paper to the company management and decision-makers are to enhance economic cooperation technical exchanges, to improve the product quality and develop new products.

On the other hand, a DSS of quality forecast evaluation will provide the standard service high quality for scientists, private groups and policymakers. Within various decision support techniques and methods through the certification procedure adopting additionally data warehouse approach can improve a product quality. In order to meet the needs of the new stage, we must shift the focus of our work to DSS and to the improvement of product quality[5]. Quality forecast efforts in the manufacturing sector have received much attention by researchers and results achieved by many companies. Thus, the manufacturer shall establish and maintain a documented forecast system to provide early warning of products quality problems and for inputting into corrective and preventive action system in production design process. Companies must concentrate our efforts on improving the quality of their products by using a DSS[6]. The department reports and publishes the results of products quality measurements each day and forecast system provides daily information to the policy-maker's decision. And the decision makers can closely communicate with the quality control department to find solution to improve quality. Moreover, the product quality can be strictly supervised and controlled by the DSS. The record of measurement and monitoring of products can be 
carried out as per control procedure for quality record based on data warehouse. Thus, some managers brought forth some sparkling new suggestions for improving product quality based on information technology. So, the excellent companies always pay close attention to perfect quality and DSS, uninterruptedly develop the product quality, research and develop the new products and new information technology, so as to meet the competitive market demand.

To date, the few papers dealing with the subject of quality forecast evaluation, regardless of mode, have largely tended to be anecdotal, with little or no empirical data to accompany the discussions. This paper presents information regarding various characteristics and design elements of the successful and highly valued DSS of quality forecast evaluation for complex products based on data warehouse. Thereby, the structures of the thesis are as follows: implementation framework for DSS based on data warehouse in the first chapter. And data warehouse data mining theory was introduced in the next chapter. Finally, related areas in need of further research are also presented in this paper.

\section{Implementation framework for DSS Based on data warehouse}

It is beneficial to reduce production costs and improve product quality for manufacturing companies to take data warehouse as a new decision support technology. While the previous research in this area has addressed numerous aspects of general quality forecast techniques and model. This article describes the configuration of various processes in DSS based on computer network techniques, data-warehouse techniques and statistical decision methods, with discussions made on how to improve the product quality. And, products quality encompasses a number of different dimensions including customer service, administration, quality control processes, maintenance and information. This article attempted to expand the research in this area and to specifically identify strategies that have proved to be successful in the quality forecast evaluation.

A DSS of quality forecast evaluation was developed to identify quality assessment practices, design elements of the quality improvement programs, and the success these programs have for the manufacturing companies using them. The DSS is based on the analysis of the data for quality management and control process[7]. The implementation framework for DSS based on data warehouse is shown in Fig. 1.

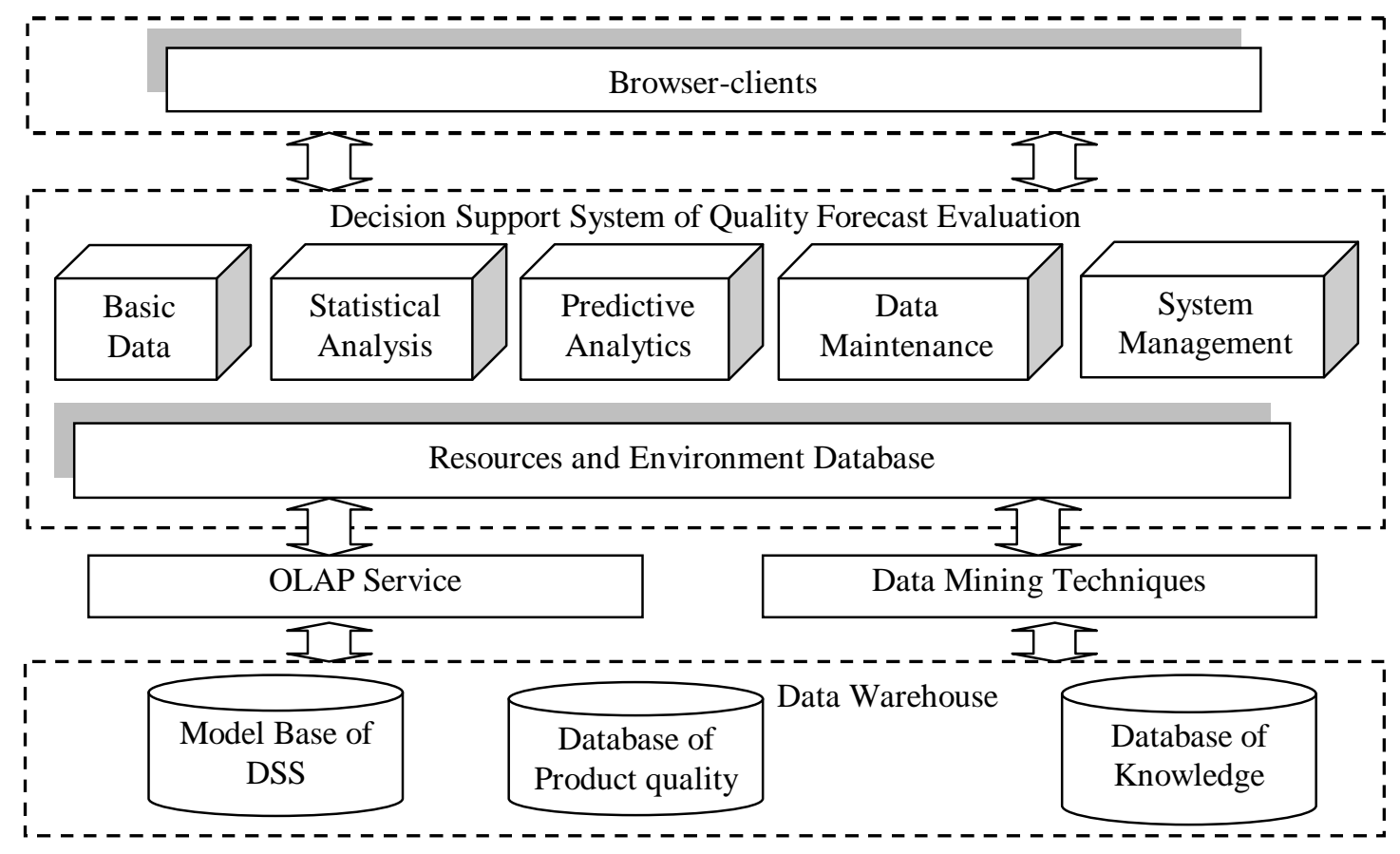

Fig. 1 The implementation framework for DSS based on data warehouse

The DSS locates original quality data and calculates all necessary quality parameters automatically. Moreover, knowledge of quality managers and frequent communications with customers both formally 
and informally is often cited as being extremely important when forecasting product quality and implementing tactics to improve quality. It is able to determine the development trend of quality management and to match these contours with the models stored in the object databases of the DSS[8]. The system is able to perform data collection, quality decision and evaluation simultaneously in real-time applications. A typical task for this system can be realized automatically, which has been solved in a number of industrial applications.

This automatic DSS minimizes the time required during installation of the system and also when changing the product handled by the system. The respondents were asked to describe the product quality of their company, based on the most recent annual quality data from data warehouse. Automated process of the system can be performed together with data warehouse[9]. Doing all the calculations for the coordinate transformation of the DSS internally, the system transmits the location data to the data warehouse[10]. A database with the functions of quality analysis and knowledge logic bank was developed for purpose of quality forecast evaluation. One main advantage of DSS over other systems is that the positioning of the sensor head for quality data collect and analysis is very flexible. Furthermore, it contains a full set of functions which are necessary in a production environment and provides many functions for product quality test and verification.

The following sections discuss the respondents' general focus on quality, customer involvement in quality forecast evaluation process, design elements of the quality programs, and finally, an implementation framework of quality forecast evaluation.

The paper proposed a quality forecast method of linear return forecast.

Using the time-sequence mean and the regression forecast mean, it was established that the forecast model of product quality, with the data warehouse from the DSS. And the process of forecasting was introduced in the following.

The elasticity coefficient is given as:

$E=\frac{\Delta y}{y} / \frac{\Delta x}{x}=\frac{\Delta y}{\Delta x} \times \frac{y}{x}$.

Then, the output of quality prediction model is defined as :

$O_{t}=O_{0}\left(1+E\left(\frac{I_{t}}{I_{0}}-1\right)\right)$.

In the same way, the prediction model of times $n$ is analyzed by mathematical formula.

$O_{t}=O_{0}(1+E I)^{n}$

The key issue for using the elastic method in the product quality forecasting is the determination elasticity coefficient. The feasible rational decision-making process with the conception of elasticity coefficient is defined in the following:

$$
E=\left(\frac{1}{n} \sum_{i=1}^{n} \frac{O_{i}-O_{i-1}}{O_{i-1}}\right) /\left(\frac{1}{n} \sum_{i=1}^{n} \frac{I_{i}-I_{i-1}}{I_{i-1}}\right) \text {. }
$$

As mentioned, the DSS can economize on quality costs and improve the quality of products by using the approved regression forecast mean method. In the decision support model, decision-making process can be adjusted according to actual quality data, and the optimal decisions can be optimized according to abnormal events to reduce waste to a minimum and improve product quality.

\section{A case study}

One indicator of an emphasis on quality can be found by simply reading a mission statement of the company. Manufacturing industry best practices is another indicator of the importance placed on assessing and improving quality. This thesis puts forward to the research of web-based interactive forecasting environment based on case methods of data warehouse, and studies the forecasting environment for further. With practical case of CNC machines, this paper verifies the comprehensive forecasting and evaluation indicators system proposed. In order to continuously upgrade the product 
quality, fully adopt newer and high-level science and information technology and do our utmost to catch up with and surpass the leading data warehousing technology.

In this article, a DSS is described which cover both information technology and data warehouse applications. The construction of the DSS of quality forecast evaluation and alarm will be stepped up, complete with an observation network, a data collection and communication network, a forecast-alarm and service network and so on. Record and monitor the process parameters and product quality characteristics, which may affect the suitability and reliability of complex production quality. This paper improves forecast veracity and guides work of complex products quality forecast model. The basic data of qualified rate of products and quality costs is shown in Table 1.

Table 1 The basic data of qualified rate of products and quality costs

\begin{tabular}{c|c|c|c|c|c}
\hline $\begin{array}{c}\text { No. } \\
\text { Per hour }\end{array}$ & $\begin{array}{c}\text { Qualified rate of } \\
\text { products ( \% }) y\end{array}$ & $\begin{array}{c}\text { Quality costs } \\
\text { millions of dollar }) x\end{array}$ & $x y$ & $x x$ & $y y$ \\
\hline 1 & 94.7 & 20 & 1894.00 & 400.00 & 8968.09 \\
\hline 2 & 95.2 & 22 & 2094.40 & 484.00 & 9063.04 \\
\hline 3 & 96.3 & 27 & 2600.10 & 729.00 & 9273.69 \\
\hline 4 & 97.1 & 36 & 3495.60 & 1296.00 & 9428.41 \\
\hline 5 & 97.5 & 53 & 5167.50 & 2809.00 & 9506.25 \\
\hline 6 & 97.9 & 57 & 5580.30 & 3249.00 & 9584.41 \\
\hline 7 & 98.1 & 94 & 9221.40 & 8836.00 & 9623.61 \\
\hline 8 & 98.4 & 118 & 11611.20 & 13924.00 & 9682.56 \\
\hline 9 & 98.6 & 156 & 15381.60 & 24336.00 & 9721.96 \\
\hline 10 & 98.7 & 171 & 16877.70 & 29241.00 & 9741.69 \\
\hline
\end{tabular}

Then, we can create a scatterplot to visually assess the nature between two continuous variables of $y$ and $x$. The scatterplot for qualified rate of products and quality costs was given as shown in Fig. 2 .

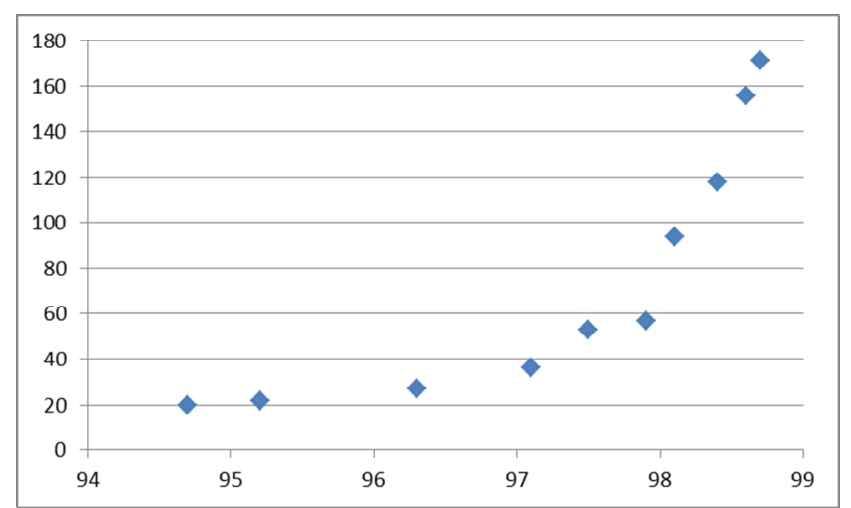

Fig. 2 The scatterplot for qualified rate of products and quality costs

Next, the implementation of DSS based on data warehouse depends on the requirements of our application. This article brings forward a scheme of DSS, which basing on data warehouse's data characteristic. The programmer can calculate and give results to model data automatically, thereby implementing the intelligence for product forecast evaluation.

For example, the elasticity coefficient can be calculated by using approved equations, which are represented as shown below.

$$
E=\left(\frac{1}{n} \sum_{i=1}^{n} \frac{O_{i}-O_{i-1}}{O_{i-1}}\right) /\left(\frac{1}{n} \sum_{i=1}^{n} \frac{I_{i}-I_{i-1}}{I_{i-1}}\right)=0.0046 / 0.2812=0.0164
$$

In the next hour, we assume that the quality costs are 182, and we will get the optimum predictive value is $98.81 \%$, which is very close to the measured value.

$$
O_{t}=O_{0}\left(1+E\left(I_{t} / I_{0}-1\right)\right)=98.7 \times(1+0.0164 \times 182 / 171-1)=98.81 \text {. }
$$

All these results showed that the company can adopt advanced information technology and equipment and scientific managerial techniques, enabling these to increase the variety of their products and improve their quality. 


\section{Conclusions}

This paper has introduced the wonderful predictive technologies of DSS, the application of DSS is an effective method that can improve product quality and reduce quality cost and benefit the manufacturing enterprises. The generative stability of DSS of quality forecast evaluation for complex products can be assured and the product quality can be advanced by information technology data-warehouse techniques. Further improvement was made in product technical services such as quality inspection, standardization, metrology and further quality forecast. We can keep on improving and providing good quality products and good services to meet the request of our customers by using various advanced forecast technology.

As in other industries, lots of efforts to improve performance and competitiveness have led manufacturing companies to design and implement comprehensive quality forecast evaluation strategies. A DSS through improved product design has cut our quality problems. In general, continued empirical research is needed in the area of formalized quality forecasting and evaluation programs and successful quality practices.

\section{Acknowledgements}

This work was supported by the National Natural Science Foundation, China (No.71301120), the Provincial Natural Science Foundation, Zhejiang, China (No.LY13G010002).

\section{References}

1. Donnelly A., Misstear B., Broderick B. Real time air quality forecasting using integrated parametric and non-parametric regression techniques, Atmospheric Environment. 103, (2015) 53-65.

2. Masoud N., Azadivar F. Design for optimal quality by recycling returned products, International Journal of Sustainable Engineering. 6(3), (2013) 267-277.

3. Naebulharam R., Zhang L. Bernoulli serial lines with deteriorating product quality: Performance evaluation and system-theoretic properties, International Journal of Production Research. 52(5), (2014) 1479-1494.

4. Qattawi A., Mayyas A., Abdelhamid M., Omar M. A. Incorporating quality function deployment and analytical hierarchy process in a knowledge-based system for automotive production line design, International Journal of Computer Integrated Manufacturing. 26(9), (2013) 839-856.

5. Kodogiannis V. S., Petrounias I., Lygouras J. An intelligent based decision support system for the detection of meat spoilage, Advances in Intelligent Systems and Computing. 323, (2015) 303-310.

6. Mbgwa G. w., Prager S. D., Krall J. M. Utilization of spatial decision support systems decision-making in dryland agriculture: A Tifton burclover case study, Computers and Electronics in Agriculture. 118, (2015) 215-224.

7. Swobodzinski M., Jankowski P. Evaluating user interaction with a web-based group decision support system: A comparison between two clustering methods, Decision Support Systems. 77, (2015) 148-157.

8. Tawfik H., Anya O. Evaluating practice-centered awareness in cross-boundary telehealth decision support systems, Telematics and Informatics. 32(3), (2015) 486-503.

9. Suri B., Singh P. Metrics for data warehouse quality, Lecture Notes in Electrical Engineering. 312, (2015) 389-396.

10. Gosain A., Singh J. Quality metrics for data warehouse multidimensional models with focus on dimension hierarchy sharing, Advances in Intelligent Systems and Computing. 320, (2015) 429-443. 